\title{
Energy Efficient Wireless Networking of Sensor Nodes
}

\author{
Ilker Demirkol $^{1,2}$, Josep Paradells ${ }^{1,2}$, Joaquim Oller ${ }^{1,2}$, Jordi Casademont ${ }^{1,2}$, Anna Calveras ${ }^{1,2}$, Marisa $^{2}$ \\ Catalan $^{2}$ \\ ${ }^{1}$ Department of Telematics Engineering, Universitat Politecnica de Catalunya \\ C/ Jordi Girona, 1, 08034, Barcelona, Spain \\ ${ }^{2}$ i2CAT Foundation \\ C/ Gran Capita 2-4, Edifici Nexus, 08034, Barcelona, Spain \\ \{ilker.demirkol, josep.paradells, joaquim.oller, jordi.casademont, anna.calveras\}@entel.upc.edu, \\ marisa.catalan@i2cat.net
}

\begin{abstract}
:
Wireless sensor networks have paved the way to a new era in user applications, where information can be sensed, processed, and distributed from remote environments with minimal or no human interaction. As the sensor nodes in these networks are often battery-operated and communicate wirelessly, they are constrained by limited resources such as bandwidth, processing speed and especially by energy. Therefore, depending on the user application, different challenges are encountered for the realization of wireless sensor networks. In this paper, we will present several reallife applications along with the challenges encountered and benefits provided by the networking of the sensors for these applications.
\end{abstract}

Key words: Sensor networks, wireless networking, Internet of Things, energy-efficient networking, smart cities.

\section{Introduction}

As the sensors are being incorporated into more and more applications every day, the dissemination of the sensory data wirelessly is becoming the de-facto standard for these applications. In this paper, we present several studies carried out by the Wireless Networks Group of Universitat Politecnica de Catalunya that investigates wireless networking of sensor nodes for different applications, by pointing out the challenges and benefits of the use of such networks in the corresponding applications.

One challenge of sensor networks is to achieve energy-efficient communication, which is necessary to reduce the maintenance cost and to extend the lifetime of the battery powered sensor networks. Moreover, for mains-powered sensor systems, energy efficiency in communication enables significant reductions in the total electricity need, which is important for the scalability of such systems. For example, in the user applications, such as those related to the smart cities, a profuse of sensor nodes will be in use. A small amount of improvement in energy consumption will result in high energy savings, overall.

\section{Wake-up Radio based Sensor Communication for Smart City Applications}

The low-power working modes of the recent radio chips enable current consumptions in the order of $\mu \mathrm{A}$. These low-power modes can be exploited by employing duty-cycles, where the device's radio and microprocessor periodically switch from the active mode to low-power states, where the radio is turned off and the microprocessor functionalities are limited. Even though the duty-cycle strategy achieves a remarkable improvement in terms of energy consumption, devices still have to monitor the communication channel regularly for data destined to them, which often results in idle listening, where the devices listen to the channel although there is no communication in the channel or in overhearing, where the devices listen to the communication that is not destined to them. However, both aforementioned situations result in energy waste, which is an important issue for energyconstrained devices such as sensor nodes. Another disadvantage of using duty-cycled operation is the sleeping delay, which is the time between the transmitting node has data to send to a receiver node and the instance the receiver node wakes up and starts listening to the channel. 
A promising alternative approach for low-power wireless communications is Wake-up Radio (WuR), which enables a pure asynchronous, on-demand communication mechanism, while providing an approach that can drastically reduce the aforementioned types of energy wastes. By attaching a secondary ultra-low power receiver to a wireless device, the main MCU can be switched to its lowest power mode and the transceiver can be turned off completely. They are activated only when required by an interrupt generated due to a special Radio Frequency (RF) signal sent from another node. With such a radio-triggered circuit, nodes stay asleep until their intervention is really required.

The S $\mu A-W u R x$ (Sub-1 $\mu A$ WuR receiver) [1] developed by our group implements a simple $868 \mathrm{MHz}$ wake-up circuit composed of an impedance matching network and a rectifier for this purpose. Because the collected RF energy at the antenna is very weak, a multistage voltage multiplier (VM) schema is employed. After several microseconds, such signal is suitable to be employed as an interruption in an input pin of a microcontroller. A digital comparator is placed at the end of the schema, being the only active component in the system, with a current consumption of 350nA. The comparator's threshold voltage is extracted from the voltage divider, which in turn consumes $526 \mathrm{nA}$. This WuR solution is observed to achieve wake-up activations as far as 13.5 meters with generic $868 \mathrm{MHz} \mathrm{RF}$ transmitters. Fig. 1 depicts the WuR receiver developed and attached to a WSN mote developed within our group [3].

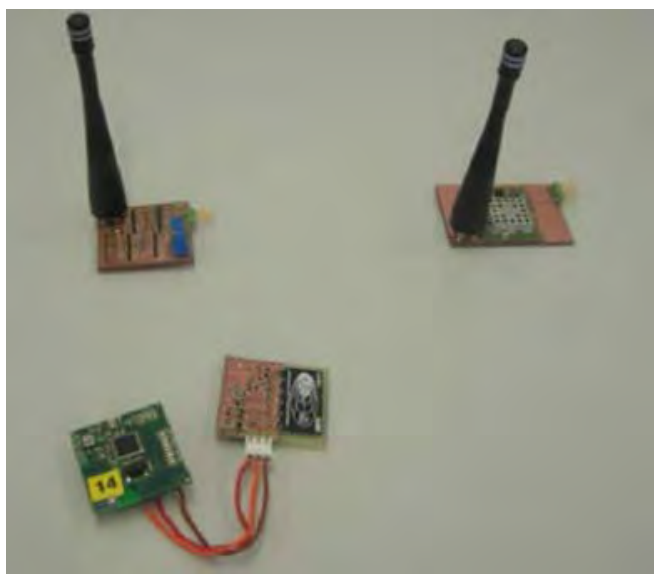

Fig. 1. The $868 \mathrm{MHz}$ WuR receivers (WuRx) attached to a WSN mote.

This WuR system is developed with urban monitoring applications in mind, where mobile data collectors equipped with WuR transmitter (WuTx) continuously transmit wake-up calls to wake up the sensor nodes in their vicinity, which are equipped with a WuRx. Addressing of the receiver nodes can be achieved with several methods, which are proposed and investigated in [2].

As the mobile data collector, it is possible to use the vehicles used for urban services such as garbage trucks or public transport buses to collect information of different type, such as temperature values, environmental noise or problems on the street lighting, etc.

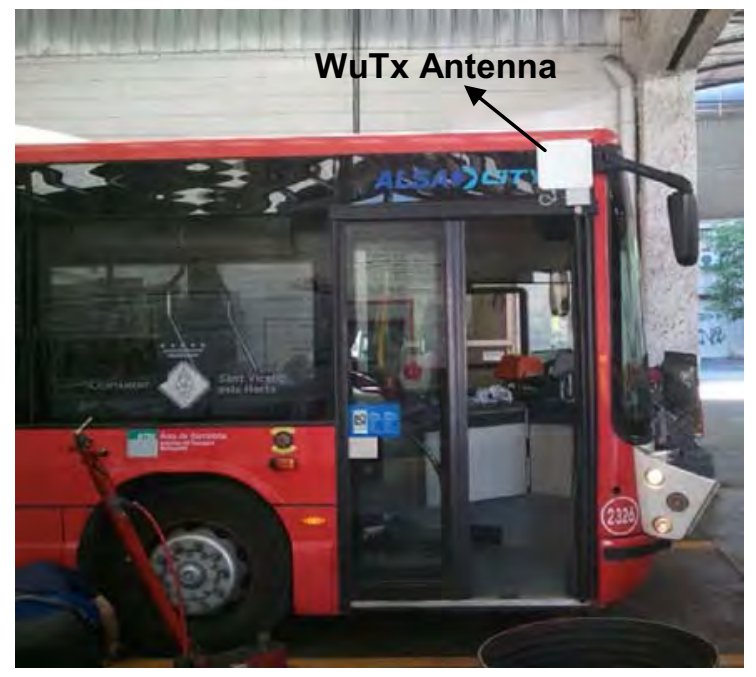

Fig. 2. Public bus equipped with WuR transmitter (WuTx).

The picture shown in Fig. 2 depicts the public bus equipped with a WuTx, which is used to collect the sensory information all along its route. Fig. 3, on the other hand, illustrates the sensory data collected from a sensor on its route. One challenge of such applications is the speed of the mobile collector, which is tackled by putting the sensors to the points where the mobile is expected to stop or slow down.

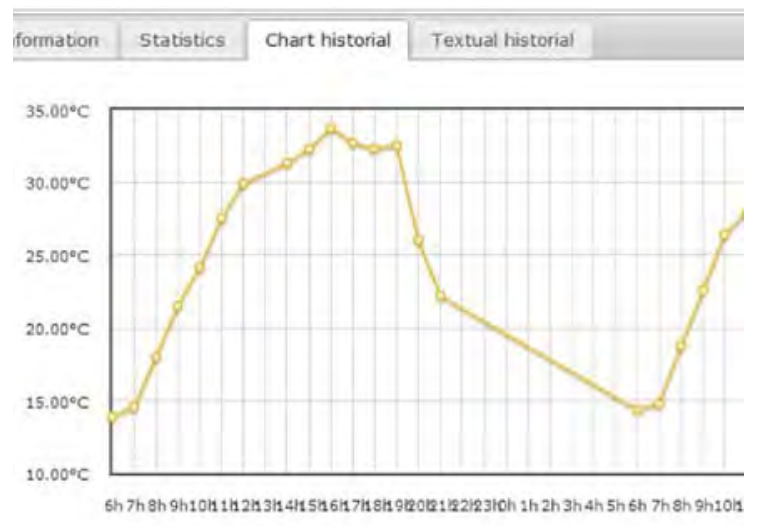

Fig. 3. Collected temperature information from an urban sensor.

Such a WuR-based smart city solutions can also be interesting to complement traditional networks (based on IEEE802.15.4 or Wi-Fi) to 
cover areas where coverage is not available or not profitable to have, or temporarily used while an infrastructure is being set up. It is also possible to propose a hybrid solution between M2M technologies for isolated sensors and / or those requiring a quick response, combined with the DTN.

\section{Sensor Network for Train Vibration Monitoring}

An interesting application that incorporates sensors and their networking is the train vibration monitoring application. The target of this application is to monitor if any train component is producing a highly irregular vibration which would indicate an early warning for a much bigger problem. Fig. 4 illustrates an example layout of the system. There are four different parts in the developed system:

1) A wireless sensor network (WSN), which acquires temperature and vibration data from train wheels and gearbox and transmits this information through a multihop trunk wireless network to a central gateway board. A sensor gateway acts as a bridge between the sensor network and the gateway board.

2) A gateway board, which manages the configuration of the sensor network and data acquisition process. It also processes received data, calculates the Fast Fourier Transform of received vibration data, generates real time alarms when it is required, compresses and stores data, and sends it to a remote central receiver via UMTS, GPRS or WiFi depending on which network is available.

3) A central server, which compresses received data and stores it into a database. It also manages alarms generated in the gateway board and calculates Fast Fourier Transform in the user side.

4) A server that allows the visualization and recovery of data from the database via a web interface.

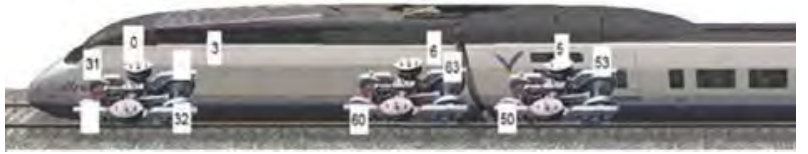

Fig. 4. Test layout. Allocation of relay and sensor nodes.

A challenge for such systems is the large data produced by the sensors. Although certain data compression methods and duty-cycling are applied, the battery life of the sensor nodes is observed to be limited. For the evaluation of battery lifetime, we performed tests by collecting the battery levels of the nodes.
From the battery data collected by the system, we can estimate the evolution of the battery in the nodes and their lifetime. There were three nodes transmitting in the gearbox (nodes 10, 12 and 13). Data only was collected when the train (and consequently) the sensor boards, were supplied. Hence, the periods without data correspond to periods when the train was not powered up. Although the manufacturer warns that nodes can stop working when their battery is lower than 2.7 volts for the employed nodes with $3 \mathrm{~V}$ batteries, we obtained data from periods where the voltage was lower.

Some test results are shown in Fig. 5. The temperature and the battery level data were generated every minute, and the vibration data was sent each time the train speed is above a specific speed. Battery evolution of node 12 can be considered typical: voltage begins to decrease slowly but when it reaches a threshold, it decreases quicker. Node 10 stops to work earlier than the others. Node 13 presents too many fluctuations. Its battery evolution is not as expected and fluctuates heavily. Its battery was depleted at the end of third day.

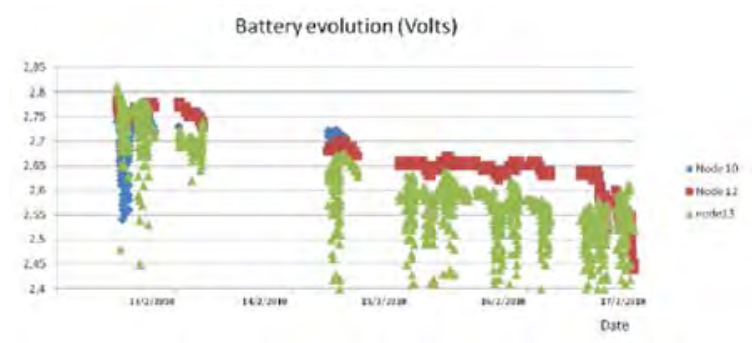

Fig. 5. Measured battery levels of the sensor nodes of a train vibration monitoring system.

Other challenges also accompany such applications. For example, in order to be sure that this sensor network system would not interfere on already installed train systems, we measured the radio spectrum generated by the sensor network using a spectrum analyser. The main conclusion was that power level generated by the sensor network is very low, and is kept inside the margins established by IEEE 802.15.4 standard [4] on the ISM band of $2.4 \mathrm{GHz}$. The values measured lead to the conclusion that this interference is even less harmful than the interference produced by other common technologies such as Wi-Fi or Bluetooth, since IEEE 802.15.4 occupies less bandwidth and uses lower transmission powers.

On the other hand, in order to characterize the scenario for which the sensor network is intended, we measured the radio spectrum generated by the train systems, or produced by other wireless devices that could affect sensor's 
transmissions. Those measurements led us to the conclusion that during the train's journey, the $2.4 \mathrm{GHz}$ ISM band is utilized by other systems, mainly due to passenger's wireless devices (Wi-Fi, Bluetooth, etc.). Moreover, we found out that when the train is moving, the interference observed is higher than the case when the train engines are on, yet the train is not moving. This resulted in changes in the topology of the sensor network during train's move, and higher packet drop ratios.

However, the developed system is shown to be stable and persistent, and achieves the objectives of the targeted application. This system is up and running in more than 40 trains as of end of 2012 .

\section{Sensor Network for Bicycle Race Positioning Application}

This project targeted a real-time and distributed tracking system of the location of people or objects involved (for example, participants in a bike race), so that this information can be used in representation and visualization of the events. For such applications, the following objectives should be met: Accuracy, small footprint and light weight product, the lack of need for additional infrastructure.

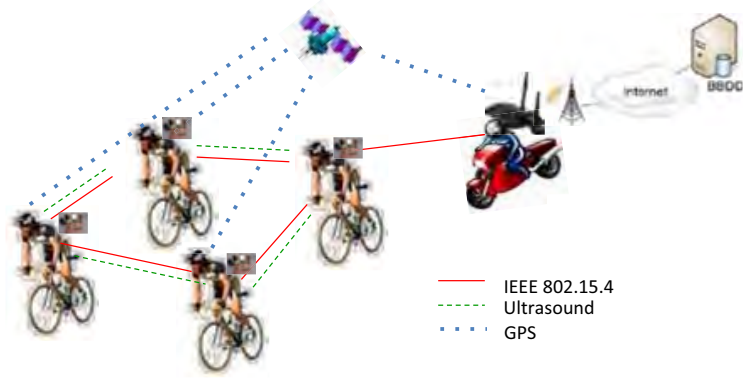

Fig. 6. System architecture of race tracking system.

The following positioning system allows realtime location of people or objects with an accuracy significantly better than the GPS system with a refresh rate of the location information of 1 second. This system is based on the communication between various network elements and which are presented in the following architecture.

Node locators are used to locate people or objects. They communicate with each other through an IEEE 802.15.4 radio interface. Each of them has an absolute GPS location and providing an ultrasound system for determining relative distances to other network devices. Each locator device is associated with a gateway.

The system also includes gateway devices which have a GPS, an IEEE 802.15.4 module and $3 G$ connectivity. Each gateway is able to collect data from up to 40 devices and transmits them to a remote server that acts as data manager. On the other hand, data management system consists of a central server connected to the Internet. Positioning data from participants are received, processed and stored to be consulted during the visual representation of the event in this system.

The message exchange protocol between different parts of the system is as follows:

- Nodes periodically update their location based on their GPS receiver (absolute location) and radio messages that are synchronized with ultrasound pulses to its neighbors (nodes up to a distance of 5 meters) can estimate how far away they are.

- Gateways periodically request location information (absolute position and distance to neighboring nodes) from the nodes. This information is received via the interface and forwarded to the IEEE 802.15.4 centralized management system interface for GPRS / UMTS.

- The data management system processes the location information and provides an estimate of position of all participants is stored in the database.

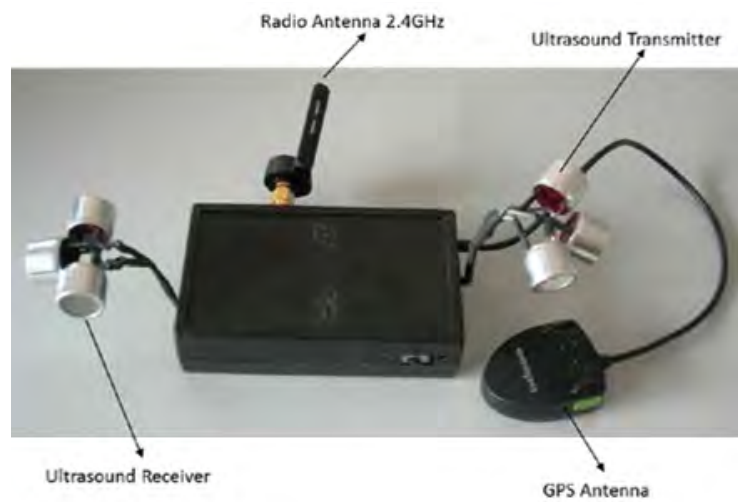

Fig. 7. Node locator device.

An important challenge in this system is to have a small footprint and lightweight solution that provides all the functionalities needed, such as GPS reception, IEEE 802.15.4 communication, ultrasound sensors, and a rechargeable battery. We could fit within a 200 grams final product shown in Fig. 7, a rechargeable Li-On Polymer $3.7 \mathrm{~V}$ battery, ultrasound sensors of $5 \mathrm{~m}$ range, an IEEE 802.15.4 module, a GPS module, and voltage divider for different components of the system.

The ultrasound transmitter modulates the 40 $\mathrm{kHz}$ pulse generated by the microprocessor. This pulse has a duration of 500 microseconds and is synchronized with a radio signal. The 
receiving device is able to determine its distance from the transmitter device by calculating the time difference between the two signals. The ultrasound transmitter circuit can operate with 3.3 to 15 volts, while feeding finally has been decided to be twice the battery voltage (i.e., $\sim 7 \mathrm{~V}$ ), thereby achieving a balance between consumption and range. It was also decided to use directional transmitters (three per node and pointing in different directions, covering more than $180^{\circ}$ ), because for the same signal level, the range is much higher than the omnidirectional transmitters which is insufficient to meet the application requirements. The physical tests in the laboratory have shown an accuracy of up to 2-3 $\mathrm{cm}$, whereas further field tests revealed an accuracy of up to $10 \mathrm{~cm}$ with these ultrasound sensors.

The GPS module used is fed with $3.3 \mathrm{~V}$ and accessed through UART interface. Its current consumption is found to be $23 \mathrm{~mA}$. The overall system has a battery life of 8 hours.

\section{References}

[1] J. Oller, I. Demirkol, J. Casademont, J. Paradells, Design, Development and Performance

Evaluation of a Low-Cost, Low-Power Wake-Up Radio System for Wireless Sensor Networks, Accepted to ACM Transaction on Sensor Networks (2012).

[2] J. Oller, I. Demirkol, J. Casademont, J. Paradells, W. Heinzelman, Time-Knocking: A Novel Addressing Mechanism for Wake-up Receivers, 8th IEEE International Conference on Wireless and Mobile Computing, Networking and Communications, IEEE WiMob'12,pp. 268-275, Barcelona, Spain, 08-10 Oct. 2012; doi:10.1109/WiMOB.2012.6379086

[3] J.L. Ferrer, Diseño e implementación de un módulo hardware de bajo coste para redes basadas en el estándar IEEE 802.15.4 para la Internet Futura. UPCCommons. 2010 http://upcommons.upc.edu/pfc/bitstream/2099.1/9 872/1/PCF JLFerrer Dise\%C3\%B10 e imp nod o IEEE 802.15.4 para la Internet Futura.pdf.

[4] IEEE 802.15.4: Wireless Medium Access Control (MAC) and Physical Layer (PHY) Specifications for Low-Rate Wireless Personal Area Networks (WPANs), September 2006. 